AN. MED. INTERNA (Madrid) Vol. 20, N. ${ }^{\circ} 10$, pp. 537-539, 2003

\title{
Trombosis venosa masiva con invasión cardiaca como primera manifestación de un hepatocarcinoma
}

\author{
M. CARNERO FERNÁNDEZ, L. E. MORANO AMADO, P. BODENLLE BELLO ${ }^{1}$, \\ F. CALVO IGLESIAS \\ Servicio de Medicina Interna-Infecciosas, ${ }^{1}$ Servicio de Radiología, ${ }^{2}$ Sección de \\ Cardiología. Universidad de Vigo. Vigo, Pontevedra
}

HEPATOCELLULAR INVOLVING VASCULAR STRUCTURES AND HEART: DIAGNOSIS AND TREATMENT

\begin{abstract}
RESUMEN
El hepatocarcinoma tiene tendencia a invadir estructuras vasculares. Es infrecuente, sin embargo, la afectación de las venas hepáticas y las metástasis intracardíacas. Presentamos el caso de un varón de 68 años con hepatopatía crónica por virus $\mathrm{C}$, que consulta por edema y dolor a nivel del miembro inferior izquierdo. Mediante eco doppler, se objetivó, trombosis venosa profunda a dicho nivel y el TAC tóraco-abdominal, mostró, trombosis completa de la vena cava inferior, trombosis de la vena suprahepática izquierda, un voluminoso trombo en aurícula derecha y una masa irregular en hígado. La alfafetoproteína fue de $77.046 \mathrm{ng} / \mathrm{ml}$. La biopsia del trombo de aurícula derecha estableció el diagnóstico de hepatocarcinoma. Se comenta la evolución del paciente tras la intervención quirúrgica, la frecuencia y clínica de esta complicación y las posibilidades terapéuticas en estos pacientes.
\end{abstract}

PALABRAS CLAVE: Hepatocarcinoma. Trombo tumoral. Metástasis intracardiacas.

\begin{abstract}
Hepatocellular carcinoma has a tendency to invade vascular structures. However, extension into the hepatic veins or heart is uncommon. We describe the case of a 68 years old man with chronic viral hepatitis type $C$, consulting about edema and pain in his left leg. Doppler scan showed deep venous thrombosis in that level and computed tomography of thorax and abdomen showed complete thrombosis of the inferior cava vein, thrombosis of the left suprahepatic vein, a voluminous thrombus in the right atrium and an irregular mass in the liver. Alpha-fetoprotein was 77,046 $\mathrm{ng} / \mathrm{ml}$ The biopsy of the rigth atrium thrombus demonstrated diseminated hepatocellular carcinoma.

We comment the patient progress after surgery, the incidence, clinical symptoms, and therapy options for these patients.
\end{abstract}

KEY WORDS: Hepatocellular carcinoma. Tumor thrombus. Intracardiac metastasis.

Carnero Fernández M, Morano Amado LE, Bodenlle Bello P, Calvo Iglesias F. Trombosis venosa masiva con invasión cardiaca, como primera manifestación de un hepatocarcinoma. An Med Interna (Madrid) 2003; 20: 537-539.

\section{INTRODUCCIÓN}

El carcinoma hepatocelular tiene tendencia a invadir las venas hepáticas (y a través de ellas, puede extenderse al lado derecho del corazón) así como la vena porta, en sus estadíos avanzados (1). Esta invasión vascular puede, no obstante, ser una complicación temprana que no indica necesariamente inoperabilidad del tumor, lo que pone de manifiesto la importancia de su diagnóstico (2). La incidencia de trombosis o afectación vascular es mucho más frecuente $(13 \%)$ en casos de hepatocarcinoma que en tumores metastásicos $(5 \%)$ o cirrosis $(5 \%)(2)$.

En general, existe una clara asociación entre tromboembolismo venoso y cáncer, sobre todo en pacientes mayores de 40 años. No obstante, sólo está justificado un screening invasivo de tumor primario ante la presencia de algún signo guía (3).
Exponemos el caso de un paciente con carcinoma hepatocelular, trombosis venosa masiva y extensión a cavidades cardiacas derechas, comentando las técnicas diagnósticas utilizadas, tratamiento y evolución.

\section{CASO APORTADO}

Varón de 68 años de edad, sin antecedentes patológicos de interés, que consulta por edema y dolor a nivel de miembro inferior izquierdo. El paciente había sido estudiado ambulatoriamente por náuseas y dolor epigástrico detectándose en la analítica sanguínea aumento de transaminasas y serología positiva para hepatitis C. A la exploración física, el paciente presentaba buen estado general, ictericia conjuntival e ingurgitación venosa yugular. A nivel abdominal, existía circulación colateral sin semiología de ascitis y hepatomegalia de 2-3 cm. El miembro inferior izquierdo estaba edematoso y el

Trabajo aceptado: 28 de febrero de 2003 
resto de la exploración era normal. En la analítica destacaba trombopenia de $62.000 / \mathrm{mm}^{3}$, dímero D 0.8-1.6 $\mu \mathrm{g} / \mathrm{ml}$, bilirrubina total 2,5 $\mathrm{mg} / \mathrm{dl}$, AST $49 \mathrm{U} / \mathrm{L}$, GGT $174 \mathrm{U} / \mathrm{L}$, fosfatasa alcalina $494 \mathrm{U} / \mathrm{L}$ y alfafetoproteína $77.046 \mathrm{ng} / \mathrm{ml}$. La radiografía de tórax mostraba mínima elevación del hemidiafragma derecho. En la ecografía abdominal, se observaba hepatomegalia, trombosis de la vena cava a nivel intrahepático, mala visualización de las venas suprahepáticas media e izquierda sugestiva de trombosis. El eco Doppler del miembro inferior izquierdo mostraba trombosis del sistema venoso profundo. En la tomografía axial computarizada (TAC) torácica y abdominal, se visualizaba trombosis completa de la vena cava inferior, trombosis de la vena suprahepática izquierda y un voluminoso trombo en la aurícula derecha con hepatomegalia de contornos nodulares compatible con hepatopatía crónica y una masa de morfología irregular, de 3 x 2,5 cm, en segmento IV-B hepático (Fig.1). El ecocardiograma mostró una masa que ocupaba la totalidad de la aurícula derecha, que protruía hacia el ventrículo derecho (Fig. 2). Se realizó resonancia magnética nuclear (RMN) cardiaca y angiorresonancia de abdomen observándose también la masa intracardiaca, trombosis parcial extensa de la vena cava inferior y trombosis tumoral de la vena suprahepática izquierda.

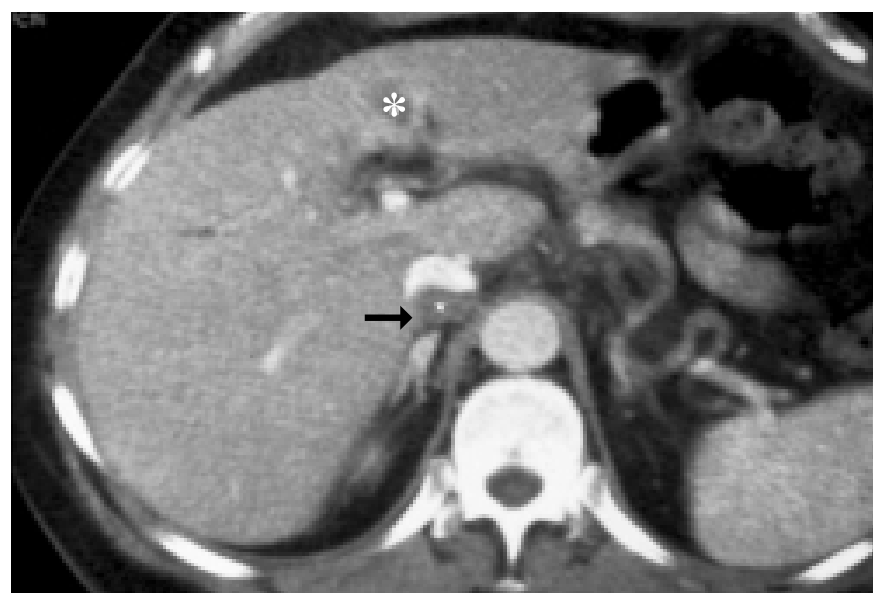

Fig. 1. TAC con contraste en fase portal. Hepatocarcinoma en lóbulo hepático izquierdo $\left(^{*}\right)$ y trombo en vena cava intrahepática $(\rightarrow)$.

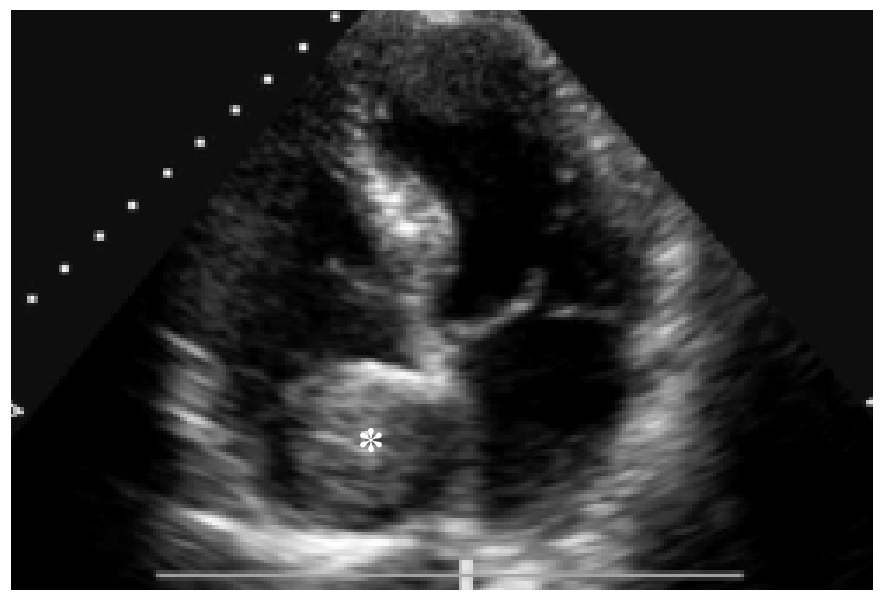

Fig. 2. Ecocardiograma con trombo en aurícula derecha (*).

\section{EVOLUCIÓN}

El paciente fue intervenido quirúrgicamente, realizándose extirpación de la masa auricular y parcialmente del trombo de las venas suprahepáticas además de trombectomía de vena cava inferior. La biopsia del trombo de aurícula fue diagnóstica de carcinoma con intensa positividad para la tinción de alfafetoproteína, siendo el diagnóstico de hepatocarcinoma. En el postoperatorio, el paciente evolucionó favorablemente, se instauró anticoagulación oral con dicumarínicos y se procedió a su alta hospitalaria. A los tres meses, se realizo TAC de control, objetivándose a nivel de ambos parénquimas pulmonares múltiples imágenes nodulares compatibles con metástasis y recidiva de la masa auricular extirpada con anterioridad. Finalmente, a los ocho meses del diagnóstico, el paciente falleció, en el contexto de un cuadro de insuficiencia hepática.

\section{DISCUSIÓN}

El hepatocarcinoma tiene tendencia a invadir estructuras vasculares, siendo más frecuente la afectación de la vena porta e infrecuente la de las venas hepáticas. Se ha descrito igualmente la invasión metastásica de la vena cava inferior y de la aurícula derecha (4-8). En pacientes con hepatocarcinoma, las metástasis en aurícula derecha como resultado de la invasión directa y extensión a través de la vena cava inferior son extremadamente infrecuentes $(9,10)$. Se han descrito metástasis tumorales en aurícula derecha más frecuentemente en casos de sarcoma testicular, linfoma, leiomiosarcoma, carcinoma epidermoide nasofaríngeo, sarcoma de Ewing y tumor de Wilms. Una vez que el tumor invade ramas de la vena hepática, la posibilidad de encontrar metástasis extrahepáticas también aumenta (5) (metástasis pulmonares, en el caso de nuestro paciente). Las manifestaciones clínicas iniciales en casos de hepatocarcinoma con invasión vascular, suelen ser las debidas a extensión metastásica del tumor, tales como edema de miembros inferiores, ascitis y circulación colateral en tórax y abdomen, en relación a invasión tumoral de la vena cava inferior $(6,10)$, simulando las de la cirrosis hepática y haciendo difícil el diagnostico diferencial con ella. La clínica secundaria al tumor, no aparece en la mayoría de las ocasiones, hasta fases avanzadas de la enfermedad. La ictericia, por ejemplo, debida generalmente a afectación parenquimatosa difusa con insuficiencia hepatocelular, implica una fase terminal y mal pronóstico (11). La ecocardiografía bidimensional es de considerable valor en el diagnóstico de tumores metastásicos en aurícula derecha $(9,12)$. En este sentido, la vena cava inferior y la aurícula derecha deberían ser estudiadas durante la realización de una ecografía abdominal, en pacientes con sospecha de hepatocarcinoma (6). Además de la ultrasonografía, las otras pruebas diagnósticas consideradas más útiles y más comúnmente utilizadas en los últimos años para diagnóstico de extensión tumoral de hepatocarcinoma (tanto intravascular como intracardiaca) incluyen la TAC y la RMN (4). En pacientes con hepatocarcinoma y extensión intravascular/ intracardiaca, la resección tumoral y eliminación del trombo tumoral es el único tratamiento radical que puede resultar en supervivencia más o menos prolongada, aunque este tratamiento se puede aplicar únicamente a pacientes con suficiente reserva funcional hepática (13-15). La eliminación del trombo tumoral sin resección hepática también se lleva a cabo para prevenir complicaciones y/o mejorar los síntomas relacionados $(4,16)$. El pronóstico de los pacientes incluso después de resección tumoral y eliminación del trombo, es generalmente malo, debido a la elevada frecuencia de metástasis a distancia, como sucedió en nuestro paciente. Por otro lado, el pronóstico de 
pacientes con trombo tumoral en la vena porta además de en la vena hepática es peor que si hay únicamente invasión de ésta última, probablemente por el alto riesgo de metástasis intrahepáticas a través de la vena porta $(4,8)$. En algunos pacientes, debido al avanzado estado de la enfermedad hepática, sólo se

\section{Bibliografía}

1. Masaki N, Hayashi S, Maruyama T, Okabe H, Matsukawa M, Unno J, Maekawa S, et al. Marked clinical improvement in patients with hepatocellular carcinoma by surgical removal of extended tumor mass in right atrium and pulmonary arteries. Cancer Chemother Pharmacol 1994; 33 (Suppl.): S7-S11.

2. Jackson V, Martin-Simmerman P, Becker G, Holden R. Real-time ultrasonographic demonstration of vascular invasion by hepatocellular carcinoma. J Ultrasound Med 1983; 2: 277-80.

3. Rodríguez A, Rodríguez R., Estelles F. Trombosis venosa profunda y cáncer. Actitud diagnóstica. An Med Interna (Madrid) 2002; 19 (12): 61-2.

4. Okada S. How to manage hepatic vein tumour thrombus in hepatocellular carcinoma. J Gastroenterol Hepatol 2000; 15: 346-8.

5. Choi B. Vascular invasion by hepatocellular carcinoma. Abdom Imaging 1995; 20 (3): 277-8.

6. Kanematsu M, Imaeda T, Minowa H, Yamawaki Y, Mochizuki R, Goto H, Seki M, et al. Hepatocellular carcinoma with tumor thrombus in the inferior vena cava and right atrium. Abdom Imaging 1994; 19: 313-6.

7. Ohwada S, Tanahashi Y, Kawashima Y, Satoh Y, Nakamura S, Kobayashi I, Ohya T, et al. Sugery for tumor thrombi in the right atrium and inferior vena cava of patients with recurrent hepatocellular carcinoma. Hepatogastroenterology 1994; 41: 154-7.

8. Nonami T, Nakao A, Harada A, Kaneko T, Kurokawa T, Takagi H. Hepatic resection for hepatocellular carcinoma with a tumor thrombus extending to inferior vena cava. Hepatogastroenterology 1997; 44: 798-802. puede llevar a cabo tratamientos paliativos (embolización arterial, quimioterapia, radioterapia), con escaso beneficio clínico. Probablemente el desarrollo de tratamientos multidisciplinarios, junto con la resección quirúrgica del tumor, logre en un futuro una mayor supervivencia de estos pacientes $(1,7)$.

9. Chia B, Choo M, Tan L, Tan A, Oon C, Chew P. Two-dimensional echocardiographic abnormalities of right atrial metastatic tumors in hepatoma. Chest 1985; 87: 399-401.

10. Kato Y, Tanaka N, Kobayashi K, Ikeda T, Hattori N, Nonomura A Growth of hepatocellular carcinoma into the right atrium. Report of five cases. Ann Intern Med 1983; 99: 472-4.

11. Diaz-Ruiz M, Falcó J, Martin J, Bella R, Carrasco M, Tortajada L. Hepatocellular carcinoma presenting as portal thrombosis with intrabiliary growth: US and MR findings. Abdom Imaging 2000 ; 25: 263-5.

12. Boiskin I, Marsan R, Boles M, Jacobs W, Demos T. Hepatocellular carcinoma with intraatrial extension. Cardiovasc Intervent Radiol 1997; 20: 324-7.

13. Fujisaki M, Kurihara E, Kikuchi K, Nishikawa K, Uemaysu Y. Hepatocellular carcinoma with tumor thrombus extending into the right atrium: report of a successful resection with the use of cardiopulmonary bypass. Surgery 1991; 109: 214-9.

14. Hamazaki K, Yagi T, Katsuta K, Edahiro T, Inagaki M, Tanaka N, Mimura $\mathrm{H}$, et al. Extracorporeal circulation and hypothermic perfusion for caval tumor thrombus complicating hepatocellular carcinoma: A case report. Hepatogastroenterology 1995; 42: 282-5.

15. Iemura J, Aoshima M, Ishigami N, Kaneda T, Oba N. Surgery for hepatocellular carcinoma with tumor thrombus in the right atrium. Hepatogastroenterology 1997; 44: 824-5.

16. Kumada K, Ozawa K, Okamoto R, Takayasu T, Yamaguchi M, Yamamoto $\mathrm{Y}$, Higashiyama $\mathrm{H}$, et al. Hepatic resection for advanced hepatocellular carcinoma with removal of portal vein tumor thrombi. Surgery 1990; 108: 821-7. 